\title{
DETERMINISTIC - STOCHASTIC SUBSPACE IDENTIFICATION FOR BRIDGES
}

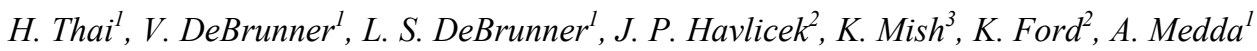 \\ ${ }^{1}$ Department of Electrical and Computer Engineering \\ Florida State University \\ ${ }^{2}$ School of Electrical and Computer Engineering \\ ${ }^{3}$ School of Civil Engineering and Environmental Science \\ University of Oklahoma
}

\begin{abstract}
System identification for structural engineering has received significant attention in the last thirty years. With the ever increasing capacity of computing technology, system identification has been applied to important structures such as bridges and aircraft. In the case of bridges, the output can easily be measured by accelerometers. Considerable research in system identification on bridges has been done using output-only models. Of course, it is difficult to measure the inputs on an in-service bridge. In this paper, we see how the inputs can be estimated from the output measurements. We then use an input-output model to develop an improved system identification technique for identifying bridges. We show that the proposed method using the estimated inputs yields superior identification in a simulated case (i.e., where everything is controlled). We then use the method on the in-service Walnut Creek Bridge located on the north-bound lanes of U.S. Interstate $\mathrm{I}-35$ between Dallas, TX and Oklahoma City, OK.
\end{abstract}

Index Terms - system identification

\section{INTRODUCTION}

System identification of large structures such as bridges, aircraft and buildings has received considerable attention. Through identification, we can determine whether the properties of the observed structures are changing over time. This is an essential component of the structural health monitoring problem. Structural engineers monitor structural response in order to detect damage. They are also interested in the modal (natural) frequencies of the system because any matching between input frequencies and modal frequencies can cause the structures to resonate, causing significant structural response with attendant damage.

Previously, the stochastic subspace identification algorithm was applied to identify bridges using an output-only model [1]. This algorithm assumes that the inputs are white processes, which is never the case. In system identification, the more information we possess and use properly, the better our produced estimation. In this paper, we will show that the deterministic-stochastic subspace identification method using an input-output system model improves the identification results over the stochastic subspace identification using the output-only system model.

\section{METHODOLOGY}

\subsection{System Modeling}

The complete system modeling algorithm is found in [2]. In this section, we only summarize the algorithmic base that is used in this paper. A bridge system under a moving load can be modeled using the following input-output state space model.

$$
\begin{aligned}
& \left\{\begin{array}{l}
x(k+1)=A x(k)+B u(k)+\omega(k) \\
y(k)=C x(k)+D u(k)+v(k)
\end{array}\right. \\
& E\left\{\left(\begin{array}{l}
\omega_{p} \\
v_{p}
\end{array}\right)\left(\begin{array}{ll}
\omega_{q}{ }^{T} & v_{q}{ }^{T}
\end{array}\right)\right\}=\left(\begin{array}{ll}
Q & S \\
S & R
\end{array}\right) \delta(p-q)
\end{aligned}
$$

where $x(k)$ is the state vector, $u(k)$ is the vector of input forces of the moving load and $y(k)$ is the vector of output measurements. The system is assumed to be stationary. The process noise $\omega(k)$ is caused by traffic, variations in the moving load, and modeling error. The measurement noise of the sensors is accounted for in the process $v(k)$. We assume $\omega(k)$ and $v(k)$ are zero mean and white sequences with the covariance given in (1).

\subsection{Algorithm}

Block Hankel matrices can be built from the input and output sequences. The input block Hankel matrix is found in equation (2), with each element being a column vector of the input sequence.

$$
U_{0 \mid 2 i-1}=\left[\begin{array}{ccccc}
u(0) & u(1) & u(2) & \cdots & u(j-1) \\
u(1) & u(2) & u(3) & \cdots & u(j) \\
\cdots & \cdots & \cdots & \cdots & \cdots \\
u(i-1) & u(i) & u(i+1) & \cdots & u(i+j-2) \\
\hline u(i) & u(i+1) & u(i+2) & \cdots & u(i+j-1) \\
\cdots & \cdots & \cdots & \cdots & \cdots \\
u(2 i-1) & u(2 i) & u(2 i+1) & \cdots & u(2 i+j-2)
\end{array}\right]=\left[\frac{U_{0 i-1}}{U_{i \mid 2 i-1}}\right]=\left[\frac{U_{p}}{U_{f}}\right]
$$

The value of $i$ must be greater than the maximum order of the system that we want to identify. The input block Hankel matrix can be divided into two parts, $U_{p}$ and $U_{f}$, which denote past and future, respectively. The output block Hankel matrix can be derived similarly. Notice that we have to choose $j$ such that $2 i+j-$ 2 is within the observed input and output sequences. The number of columns $j$ must also be much larger than the number of block rows $i$ for the algorithm to work accurately.

A combination matrix of input and output can be defined 


$$
W_{p}=\left[U_{p} \mid Y_{p}\right]^{T}
$$

An oblique projection $O b_{i}$ of the row space of $Y_{f}$ along $U_{f}$ on $W_{p}$ is computed as in (4). The oblique projection removes all of the future input information from the future output using only the past information.

$$
O b_{i}=Y_{f} /_{U_{f}} W_{p}=Y_{f}\left[\begin{array}{ll}
W_{C}^{T} & U_{f}^{T}
\end{array}\right]\left(\left[\begin{array}{cc}
W_{p} W_{C}^{T} & W_{p} U_{f}^{T} \\
U_{f} W_{C}^{T} & U_{f} U_{f}^{T}
\end{array}\right]\right)_{\text {first } r \text { columns }} \cdot W_{p}{ }^{(4)}
$$

The $\dagger$ denotes the Moore-Penrose pseudo-inverse discussed by Ben-Israel in [3]. The main deterministic-stochastic subspace identification theorem states that the oblique projection $O b_{i}$ is equal to the product of the extended observability matrix $\Gamma_{i}$ and the state sequence $X_{i}$, i.e.

$$
O b_{i}=\Gamma_{i} X_{i}=\left[\begin{array}{llll}
C & C A & \cdots & C A^{i-1}
\end{array}\right]^{T}\left[\begin{array}{llll}
x(i) & x(i+1) & \cdots & x(i+j-1)
\end{array}\right]^{(5)}
$$

The extended observability matrix and state sequence can be extracted using the singular value decomposition of $O b_{i}$.

$$
O b_{i}=U S V^{T}=\left(\begin{array}{ll}
U_{1} & U_{2}
\end{array}\right)\left(\begin{array}{cc}
S_{1} & 0 \\
0 & 0
\end{array}\right)\left(\begin{array}{c}
V_{1}^{T} \\
V_{2}^{T}
\end{array}\right)=U_{1} S_{1} V_{1}^{T}
$$

$U$ and $V$ are unitary matrices, and $S$ is a diagonal matrix; $U_{1}, V_{1}$ and $S_{1}$ are similar to $U, V$ and $S$ without their zero singular values.

The extended observability matrix $\Gamma_{i}$ is derived as following:

$$
\Gamma_{i}=U_{1} S_{1}^{1 / 2}
$$

The state sequence $X_{i}$ is the other half of the decomposition or can be calculated as in (8)

$$
X_{i}=S_{1}^{1 / 2} V_{1}^{T}
$$

The first block rows of the future block Hankel matrices can be moved into the past block Hankel matrices to yield we the Hankel matrices $U_{p}^{+}, U_{f}^{-}, Y_{p}^{+}, Y_{f}^{-}$and the input-output matrix $W_{p}^{+}$. Another oblique projection is defined.

$$
O b_{i-1}=Y_{f}^{-} /_{U_{f}^{-}} W_{p}^{+}
$$

The main theorem also holds for the new oblique projection.

$$
O b_{i-1}=\Gamma_{i-1} X_{i+1}
$$

The state sequence $X_{i}$ can be derived from the new oblique projection.

$$
X_{i+1}=\Gamma_{i}^{\dagger} O b_{i-1}
$$

The system matrices $(A, B, C, D)$ can be determined in a least square sense in the following equation system.

$$
\left(\begin{array}{c}
X_{i+1} \\
Y_{i \mid i}
\end{array}\right)=\left(\begin{array}{cc}
A & B \\
C & D
\end{array}\right)\left(\begin{array}{c}
X_{i} \\
U_{i \mid i}
\end{array}\right)+\left(\begin{array}{c}
\rho_{\omega} \\
\rho_{v}
\end{array}\right)
$$

The above system matrices are the true system matrices up to a similarity transformation.

\subsection{Input estimation}

In the case of real bridges, it is difficult to measure the inputs due to hardware and access limitations. In our experience inspecting the bridge accelerations, we have found that only traffic that significantly impacts the bridge is heavy trucks. Therefore, we propose to estimate the truck inputs using our estimated output (the easily measured bridge accelerations). In our approach, we want to find the initial position of the truck (the position of the truck when we start collecting data) and the velocity of the truck.

From the measured output accelerations, we determine that the vibration amplitudes are strongest when the vehicle is directly above the accelerometer. At other times, there are only small changes in accelerations. Consequently, a straightforward estimation process for our required inputs can be developed. First, we sum the energy from the outputs of the bridge as the truck crosses. We determine the amount of time in this observation that divides that energy into two equal portions. Now we estimate the time required by the truck to reach the sensors. Now, since we know the position of the sensors on the bridge and the time that the truck required to reach each sensor, we can determine the initial position and velocity of the truck using the least square method. The resulting estimates can now be plugged into a bridge-load model to generate the inputs required in the system identification algorithm.

\subsection{Modal Analysis}

A mode of a structure is a pattern of deformation of the structure at a specific harmonic frequency [4]. Modes are of concern to structural engineering because significant damage due to resonance can result if a structure is excited by an external force having a frequency that coincides with one of the modes. The frequencies of the structural modes can be found by taking the imaginary parts of the eigenvalues of the continuous-time state matrix $A$ [4]. These natural frequencies may be determined as

$$
f_{i}=\frac{\arg \left(\lambda_{i}\right) \cdot f_{s}}{2 \pi}
$$

where $\lambda_{i}$ is the $i^{\text {th }}$ eigenvalue of the discrete-time state matrix A, $f_{S}$ is the sampling frequency, and $f_{i}$ is in $\mathrm{Hz}$.

\subsection{Order Determination}

Theoretically, after all the zero singular values are removed from the diagonal matrix $S$, the dimension of $S_{1}$ is the order of the system. In real systems, all signals are subject to noise and so there are no identically zero singular values. It is hard to determine the correct order when noise is added to the signals. One of the tools for order determination is the stabilization diagram described in [5]. The basic idea of the stabilization diagram is that we can pick the $n$ highest singular values from the $\mathrm{S}$ matrix and compute the natural frequencies of the structure. We iterate $n$ from 2 to the maximum order that we want to identify for the structure and compare the natural frequencies of the current order with those of one order lower. If the frequencies are within a given tolerance, we will accept them as stable estimates of natural frequency. In this paper, we choose the tolerance to be $2 \%$, so that the criterion for accepting a new mode is given by

$$
\frac{f^{(n)}-f^{(n-1)}}{f^{(n)}}<2 \%
$$

Although the stabilization diagram can be used to identify and retain the physical poles, it does not show us the energy or the strength of those modes. For our purposes, we want to find the most important modes and get rid of the insignificant ones.

The whole system can be decoupled into individual modes. Each mode can be represented by a state space equation.

$$
\left\{\begin{aligned}
x(k+1) & =\lambda_{i} x(k)+b_{i} u(k) \\
y(k) & =c_{i}(k)
\end{aligned}\right.
$$

where $\lambda_{i}$ is the $i^{\text {th }}$ eigenvalue of the identified state matrix $A, b_{i}$ is the $i^{\text {th }}$ row of the input matrix $B$ and $\mathrm{c}_{\mathrm{i}}$ is the $i^{\text {th }}$ column of the output matrix $C$. $B$ and $C$ are obtained from the identified matrices $B$ and $C$ by a similarity transformation such that the 
matrix $A$ becomes a diagonal matrix. The feed-through matrix $D$ can be ignored since the inputs are the same for all the modes.

The transfer function of each mode is defined as:

$$
G_{i}(z)=\frac{c_{i} b_{i}}{z-\lambda_{i}}
$$

We compute the norms of the transfer functions of the modes to determine which modes are significant in the system. In this paper, we use the $\mathrm{H}_{2}$ norm described in [4]

$$
\left\|G_{i}\right\|=\left(\frac{1}{2 \pi} \int_{0}^{2 \pi} \operatorname{tr}\left(G_{i}^{*}\left(e^{j \theta}\right) G_{i}\left(e^{j \theta}\right)\right) d \theta\right)^{1 / 2}
$$

Where the $\operatorname{tr}()$ denotes the sum of the diagonal elements and $*$ is the conjugate transpose.

There are several approaches to determine the model order of the system. One approach is that we truncate at the point that has the biggest gap in $\mathrm{H}_{2}$ norms. The disadvantage of this method is that there may be no clear gap between the modes. A better approach appears to be based on thresholds. For example, a hard threshold of $90 \%$ would mean that we retain only those largest norm modes that contribute $90 \%$ of the total energy computed, and thus remove those smallest norm modes contributing only $10 \%$ of the total. The $90 \%$ threshold corresponds to a $9.54 \mathrm{~dB}$ "SNR" where the signal is the large norm components and the noise is the small norm components. After analyzing the identified system through the stabilization and the $\mathrm{H}_{2}$ norm, we can determine the order of the system consisting of significant modes.

\section{EXPERIMENTAL CASES}

\subsection{Bridge-Load Simulation}

An effective, practical bridge-load simulation was thoroughly described in [6]. This simulation consists of two parts: bridge simulation and load simulation. The bridge is simulated using a finite element analysis for a beam. The load is simulated by a three-force constant speed load such as would be found by a truck wheel loading. The input forces and output accelerations are collected for identification.

The true modal frequencies can be computed and compared with the results of the system identification methods. Table 1 shows the first six true modal frequencies of the bridge simulation.

\begin{tabular}{|c|c|}
\hline Mode & Frequency \\
\hline 1 & $0.8244 \mathrm{~Hz}$ \\
\hline 2 & $2.2667 \mathrm{~Hz}$ \\
\hline 3 & $4.4054 \mathrm{~Hz}$ \\
\hline 4 & $7.1289 \mathrm{~Hz}$ \\
\hline 5 & $10.1888 \mathrm{~Hz}$ \\
\hline 6 & $14.1084 \mathrm{~Hz}$ \\
\hline
\end{tabular}

Table 1: True modal frequencies of the bridge simulation

The test case in this paper has $39 \mathrm{~dB}$ input noise and $57 \mathrm{~dB}$ output noise. Fig. 1 illustrates the stabilization diagram of the bridge-load simulation using our proposed deterministicstochastic subspace identification (input-output model). We observe that it can detect the first six modes. Modes 2 and 3 are more susceptible to the noise than are the other modes. Fig. 2 illustrates the stabilization diagram of the bridge-load simulation using stochastic subspace identification (output-only model) which was used in [1]. We also use the same set of data as in Fig. 1 for a fair comparison. Modes 2 and 3 cannot be detected by this method. So, our proposed method outperforms the existing one.

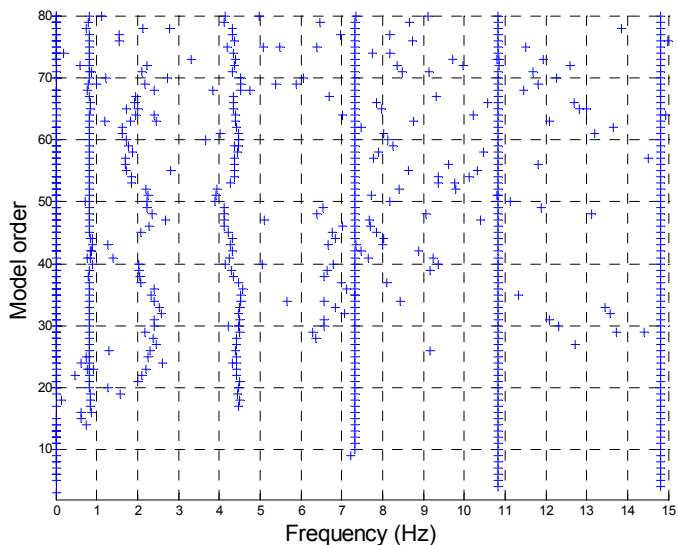

Fig. 1: Stabilization diagram using deterministic-stochastic subspace identification on bridge-load simulation.

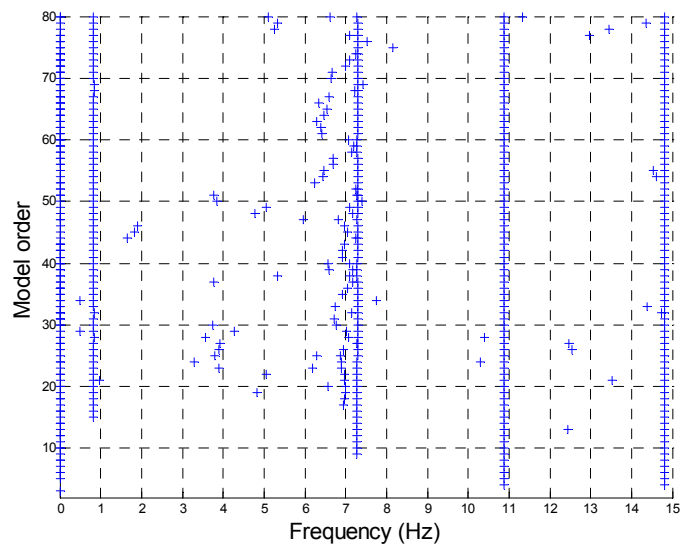

Fig. 2: Stabilization diagram using stochastic subspace identification on bridge-load simulation.

More analyses can be found in [6] which show that the stochastic subspace identification sometimes introduces spurious modes. This is explained by the fact that stochastic subspace identification assumes white noise inputs, which is rarely ever the true case in practice. Therefore, a better system model can be obtained by estimating the true system inputs.

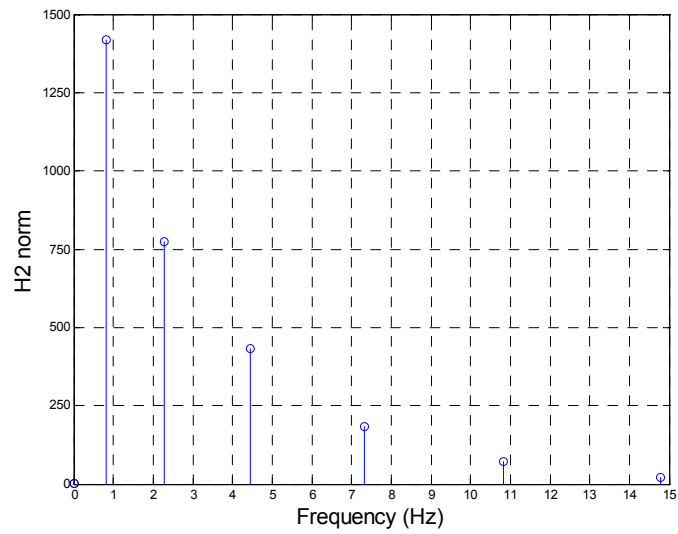

Fig. 3: $\mathrm{H}_{2}$ norms of the modes of the bridge simulation. 
The $\mathrm{H}_{2}$ norm diagram of the modes is shown in Fig. 3. The modal energies are concentrated in the lower frequency modes of the system, as expected by structural engineers.

\subsection{Walnut Creek Bridge}

Walnut Creek Bridge (Purcell, Oklahoma) is the bridge on Interstate highway I-35, near mile marker 91 in the northbound direction (see Fig. 4). The bridge is $400 \mathrm{ft}$ long. It consists of 4 spans; each span is $100 \mathrm{ft}$. long. The test span is chosen to be the third span. The outputs were collected from the accelerometers placed on the underside of the bridge along the girders. The inputs were estimated by the input estimation method discussed above. Fig. 5 illustrates the stabilization diagram after identification using our algorithm. We find 13 modes with frequencies below $10 \mathrm{~Hz}$.

\section{WanutCreek Bridge Sataisitics}

\section{Walnut Creek Bridge on 1-35 near mile marker 91 built in 1971}

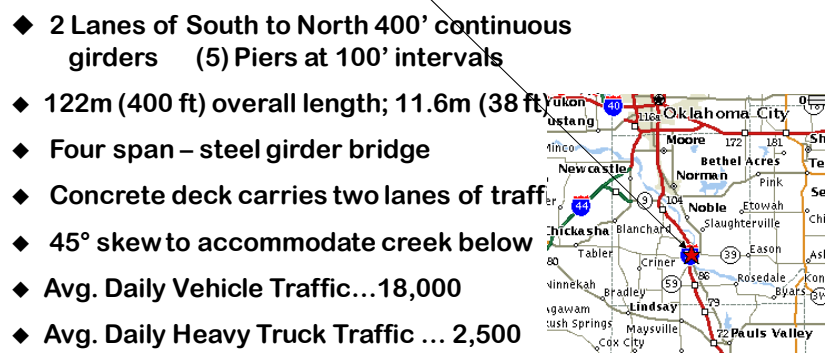

Fig. 4: Walnut Creek Bridge

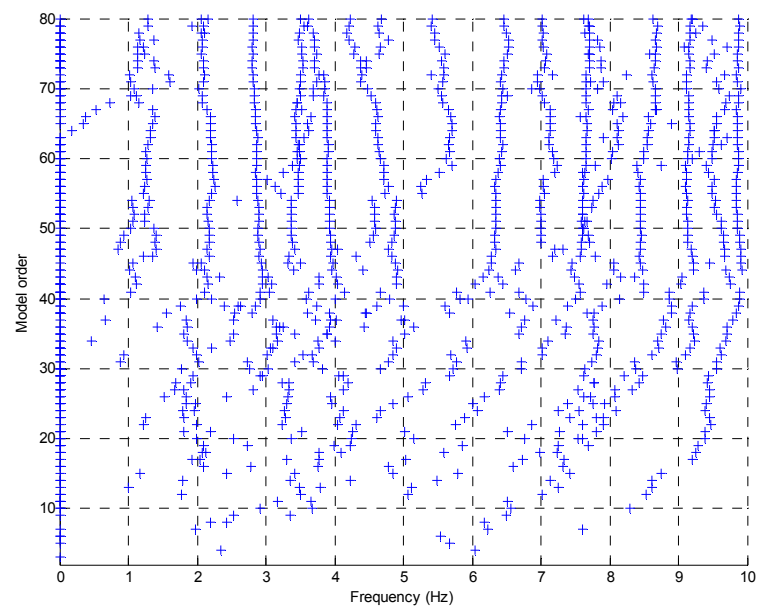

Fig. 5: Stabilization diagram using deterministic-stochastic subspace identification on Walnut Creek Bridge.

Fig. 6 shows the strength of the modes. We observe that the energy is concentrated in the low frequency modes except for the modes at $74.25 \mathrm{~Hz}$ and $101.42 \mathrm{~Hz}$.

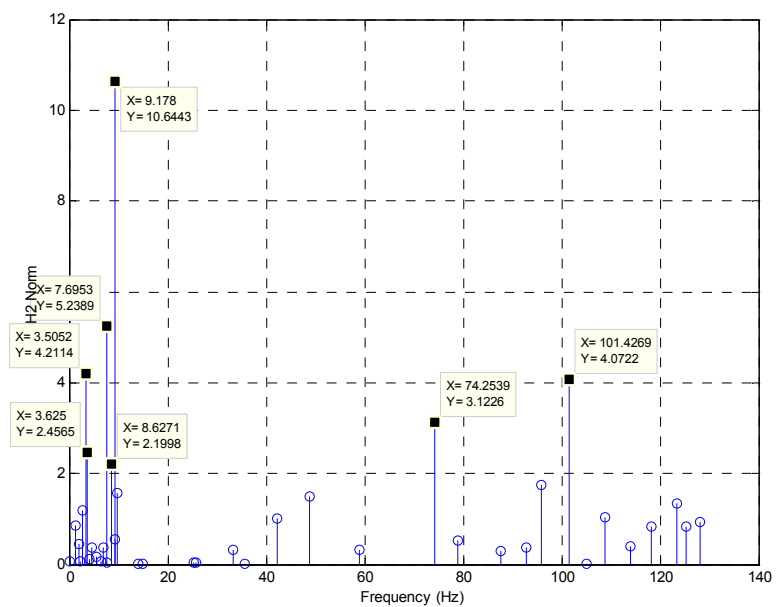

Fig. 6: $\mathrm{H}_{2}$ norms of the modes of the Walnut Creek Bridge

It is difficult to verify the results with the true modes of the bridge because the bridge has been in service for more than 40 years. Visual inspection of the bridge confirms extensive structural damage to the structure (e.g. a twisted girder is present in the observed span of the bridge). However, we can compare our results with previous (imperfect) identification on this bridge. The impulse response method [7] was applied by using a dropped hammer on the Walnut Creek Bridge in 1996, and the stochastic subspace identification method was used in 2004 [1].

\begin{tabular}{|c|c|c|}
\hline deterministic-stochastic & impulse & stochastic \\
\hline $1.2191 \mathrm{~Hz}$ & - & - \\
\hline $2.0665 \mathrm{~Hz}$ & - & - \\
\hline- & $2.500 \mathrm{~Hz}$ & - \\
\hline $2.8201 \mathrm{~Hz}$ & & $2.9579 \mathrm{~Hz}$ \\
\hline- & $3.125 \mathrm{~Hz}$ & - \\
\hline- & - & $3.3124 \mathrm{~Hz}$ \\
\hline $3.5052 \mathrm{~Hz}$ & $3.500 \mathrm{~Hz}$ & - \\
\hline $3.6250 \mathrm{~Hz}$ & $3.625 \mathrm{~Hz}$ & - \\
\hline- & $3.875 \mathrm{~Hz}$ & - \\
\hline $4.2309 \mathrm{~Hz}$ & $4.250 \mathrm{~Hz}$ & - \\
\hline- & $4.375 \mathrm{~Hz}$ & $4.3435 \mathrm{~Hz}$ \\
\hline- & $4.750 \mathrm{~Hz}$ & - \\
\hline- & $5.000 \mathrm{~Hz}$ & - \\
\hline- & - & $5.2461 \mathrm{~Hz}$ \\
\hline $5.4159 \mathrm{~Hz}$ & - & - \\
\hline- & $5.625 \mathrm{~Hz}$ & - \\
\hline $6.4455 \mathrm{~Hz}$ & - & - \\
\hline $7.0118 \mathrm{~Hz}$ & - & - \\
\hline- & - & $7.2101 \mathrm{~Hz}$ \\
\hline $7.6953 \mathrm{~Hz}$ & $7.625 \mathrm{~Hz}$ & - \\
\hline- & $8.000 \mathrm{~Hz}$ & $8.0368 \mathrm{~Hz}$ \\
\hline
\end{tabular}

Table 2: Comparison among deterministic-stochastic, stochastic subspace identification and impulse methods

Inspecting Fig. 6 and Table 2, we observe that the high energy modes in our deterministic-stochastic subspace identification match with the impulse response method (at $3.5052 \mathrm{~Hz}, 3.625 \mathrm{~Hz}$, $4.2309 \mathrm{~Hz}$ and $7.6953 \mathrm{~Hz}$ ). The stochastic subspace identification does not produce results that closely match with either of the two 
other methods. This is probably explained by the fact that that algorithm assumes the inputs are white while they are, in fact, not white. The high frequency modes that we detected can be explained by either the characteristic of the moving load or the uncertainty that was created when we estimated the inputs.

\section{BRIDGE MONITORING SYSTEM}

The Bridge Monitoring System (BMS) has been developed at the Dynamic Structures Sensing and Control (DySSC) Center at the University of Oklahoma with the intention to acquire bridge data continuously over long periods of time. The BMS has four major components: central computer, sensor prototypes, network devices and power system. The sensor nodes collect output data (mainly acceleration). The central computer acquires the data from the sensor prototypes and archives them. The network devices maintain the connection between the central computer and sensor prototypes. The power system gets energy from solar panels. It provides the power for the whole system to ensure that the BMS can operate continuously. Using the TCP/IP protocol, the BMS can theoretically handle up to four million sensors in one local network with high speed communication. The protocol also allows us to connect to the system remotely. The detailed implementation of the system is described in [6] and [8]. The system provides the real data for us to identify bridge systems. The system will also be used for other purposes such as bridge health monitoring/damage detection and real-time feedback control on moving trucks.

Currently, the system has been successfully implemented at the Walnut Creek Bridge, and a similar system with improved electronics was successfully deployed at the Canadian River Bridge on the southbound lanes of Interstate I-35 in Oklahoma (Fig. 7). A test system was also implemented at the Walnut Creek Bridge. Additional analysis from the Walnut Creek Bridge data can also be found in [6].

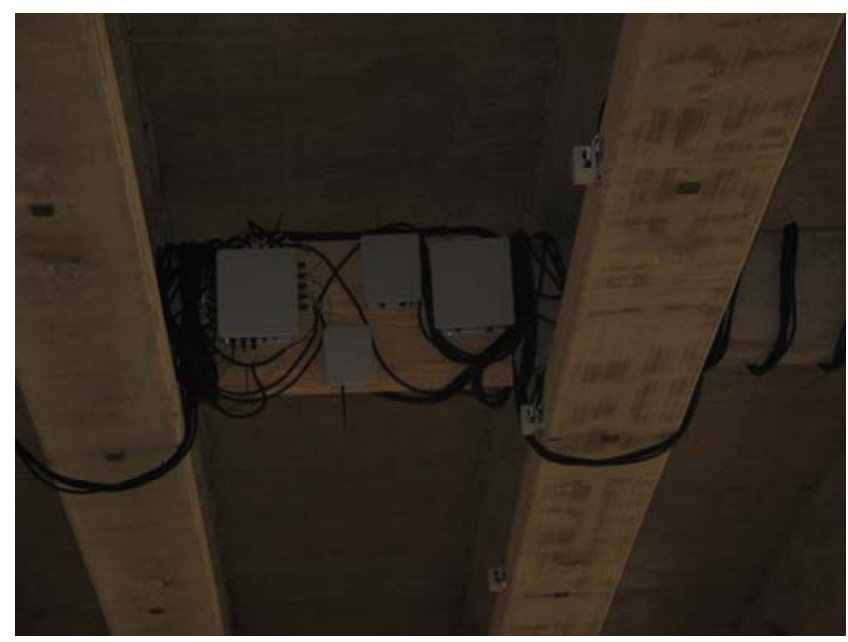

Fig. 7: The Bridge Monitoring System at Canadian River Bridge

\section{CONCLUSIONS AND FUTURE WORK}

The deterministic-stochastic subspace identification with estimated inputs significantly improves the results of identification of bridge systems compared to the stochastic output-only algorithm. Our input-output system model is able to more appropriately handle the unknown inputs compared to the stochastic algorithm that is built on the implicit erroneous assumption that the inputs are white.

The identification results can be used by structural engineers to monitor the health conditions of the bridges and detect the damage by tracking any changes in the structural modes over time. They can also be used to alter the frequencies of the truck chassis in order to attenuate any resonance of the truck chassis with the bridge.

Our identification results from Walnut Creek Bridge show several high energy modes at high frequencies which could be from the truck chassis, from estimation errors that creep into the results when we estimate the inputs of the trucks, or physically present in the structure. This must be clarified in the future.

For future research, we can incorporate the Kalman filter state into the state space model to improve the results. We can also study several bridge and truck models to have better identification results. Furthermore, measuring the initial position, velocity and weight of the trucks by hardware will help to reduce the errors on estimation of the inputs. The subspace identification also introduces the spurious mode at DC which can also be investigated.

\section{REFERENCES}

[1] V. DeBrunner, L.S. DeBrunner, P. Wang, J. David Baldwin, A. Medda and H. Thai, "Stochastic sub-space identification methods for Bridges", Proceedings of the $39^{\text {th }}$ Asilomar Conference on Signals, Systems and Computers, pp. 1153$1157,2005$.

[2] P. Van Overschee and B. De Moor, Subspace Identification for Linear Systems: Theory-Implementation-Applications, Dordrecht, The Netherlands, 1996.

[3] A. Ben-Israel, "The Moore of the Moore-Penrose inverse", Electronic Journal of Linear Algebra, vol. 9, pp. 150-157, 2002.

[4] W. K. Gawronski, Advanced Structural Dynamics and Active Control of Structures, Springer-Verlag, New York, 2004.

[5] H. Van der Auweraer and B. Peeters, "Discriminating physical poles from mathematical poles in high order systems: use and automation of the stabilization diagram", Instrumentation and Measurement Technology Conference IMTC 04, vol. 3, pp.2193-2198, 2004.

[6] H. Thai, System Identification of Bridges under a Moving Load and Implementation of the Bridge Monitoring System, Master Thesis, The School of Electrical and Computer Engineering, University of Oklahoma, 2007.

[7] J. Pang, Modeling and Experimental Modal Analysis of Highway Bridges, $\mathrm{PhD}$ Thesis, The School of Aerospace and Mechanical Engineering, University of Oklahoma, 1996.

[8] K. Ford, Computer Hardware for Vibration Mitigation and Monitoring, Master Thesis, The School of Electrical and Computer Engineering, University of Oklahoma, 2007. 